\title{
Radiographic measurement of canine stifle joint angles using four different landmark methods
}

\author{
Karol Ševčík, Marian Hluchý, Marieta Ševčíková, Valent Ledecký \\ University of Veterinary Medicine and Pharmacy in Košice, Small Animal Clinic, Košice, Slovak Republic
}

Received April 28, 2021

Accepted November 9, 2021

\begin{abstract}
This study was conducted to compare angles physically set on the stifle joints of cadaveric limbs of dogs with the results by four different radiographic methods for stifle angle measurement. Thirteen pelvic limbs from various large breeds and skeletally-mature dogs were used. The stifles were fixed at four angles: $125^{\circ}, 130^{\circ}, 135^{\circ}$ and $140^{\circ}$. Altogether 52 radiographs were done. Each stifle angle set on the cadaver limbs was radiographically measured using four sets of landmarks (the goniometric, long axis, eminence and kinematic methods). We found similarity between angles physically set on cadaver limbs and radiographically measured using the long axis method $(P>0.05)$. The goniometric method showed similarity in group of limbs with the stifle fixed at $140^{\circ}(P>0.05)$, and other measurements differed significantly $(P<0.05)$. Eminence and kinematic method measurements were different compared to the angle of fixation of the stifle on the cadaver $(P<0.05)$ but similar compared to each other $(P>0.05)$. The method of stifle joint angle measurement should be considered when comparing similar studies, and also in preoperative measurements for some tibial osteotomies aimed at stabilizing the joint after cranial cruciate ligament rupture.
\end{abstract}

\section{Determination of angulation, goniometry, stifle instability, dog}

Of all the joints in dogs, the stifle joint is most commonly affected with diseases. Most problems relate to joint instability and osteoarthritis because of degeneration and rupture of the cranial cruciate ligament (CrCL) (Vas s eur 2003). A large number of different surgical procedures have been described for the treatment of stifle instability in dogs, and decisionmaking regarding surgical treatment for $\mathrm{CrCL}$ remains controversial. Currently the most commonly used are tibial osteotomies (TO). Nisell, who described the resultant joint force as approximately parallel to the patellar ligament, based the advancement of tibial tuberosity on mechanical model analysis of the human knee (Nisell et al. 1986). Based on these data, Montavon and Tepic proposed that a similar situation exists in dogs, and tibial tuberosity advancement (TTA) would similarly neutralize cranial tibiofemoral shear force in CrCL-deficient stifle joints in dogs (Montavon et al. 2002; Tepic et al. 2002; Tepic and Montavon 2004). Based on experimental ex vivo models, it has been confirmed that if the patellar tendon angle (PTA) is approximately perpendicular, these shear forces are neutralized and the point is referred to as the "crossover point" (Apelt et al. 2007; Miller et al. 2007; Kipfer et al. 2008; Hoffmann et al. 2011).

An idealized mid-stance stifle angle of $135^{\circ}$, necessary for preoperative planning for some tibial osteotomies, has been proposed from kinematic gait studies, with well-defined anatomical landmarks used to determine the stifle angle (Allen et al. 1994; Schaefer et al. 1998; Tepic et al. 2002; Kipfer et al. 2008; Hoffmann et al. 2011). The most commonly used were as follows: the greater trochanter of the femur, the lateral malleolus of the fibula, and a point of rotation between the fibula head and the femur (Allen et al. 1994; Schaefer et al. 1998; Montavon et al. 2002). For the sake of comparison, the

Address for correspondence:

Marian Hluchý

Small Animal Clinic

University of Veterinary Medicine and Pharmacy in Kosice

Košice, Slovak Republic 
landmarks used for stifle joint angle measurement in ex vivo clinical studies are not described (Apelt et al. 2007; Miller et al. 2007; Kim et al. 2009), and in the clinical case series of TTA, the anatomical landmarks were, the long axes of the femur and tibia, it means that they were different from those described in the kinematic gait studies mentioned above (Hoffman et al. 2006; Lafaver et al. 2007; Stein and Schmoekel 2008; Voss et al. 2008). Consensus is however lacking among authors regarding the optimal landmarks for radiographic determination of the stifle angle. It would be useful to be able to accurately estimate the real stifle angle using radiographically repeatable anatomical landmarks on the pelvic limbs of dogs (Barnes and Owen 2015). Otherwise, there is still a risk of incorrect preoperative measurements for some TO (Bush et al. 2011).

The purpose of our study was to try to define radiographically repeatable anatomical landmarks which would correlate with the goniometric measurement of the stifle angle set physically on cadaver limbs. In connection with this we hypothesized that if we were able to determine the centre of the medial condyle on the radiographs using the Blumensaat's line, then the radiographic measurement might correlate with the angle set on the cadaver limbs according to the goniometer (Barnes and Owen 2015).

Our second aim was to compare the angles set physically on cadaver limbs with the angles measured using different radiographic methods (RM), and we assumed that the angles set on the cadavers would differ from those measured by means of RM, except the first one as mentioned above.

Finally, we wanted to compare individual RM with each other, and we hypothesized that the use of different landmark methods would result in different stifle angles being measured.

\section{Materials and Methods}

We used thirteen pelvic limbs from various skeletally-mature dogs weighing in excess of $25 \mathrm{~kg}$, euthanized for reasons unrelated to this study. Prior to measurement the stifle joints were radiographically evaluated for any signs of degenerative diseases, and affected ones were excluded. Each limb was harvested by disarticulation of the coxofemoral joint.

The limbs were placed in the medio-lateral view, such that the head of the femur, the stifle and the tibiotarsal joint were all positioned on the cassette. Using a standard transparent plastic goniometer, with one arm directed towards the longitudinal axis of the femur and the second arm centred over the medial malleolus of the tibia, the stifle joint angle was measured approximately at the point of the centre of the medial femoral condyle. We used the centre of the proximal and middle part of the diaphysis of the femurs because the limbs were positioned on the lateral side on the table, so we could not use the line connecting with the great trochanter as it was used by Jaegger et al. (2002). We linked these two points with the centre of the medial condyle of the femur. The stifles were physically positioned at four angles: $125^{\circ}, 130^{\circ}, 135^{\circ}$ and $140^{\circ}$. Each limb was fixed at the desired angle with intramedullary pins drilled into the proximal part of the femur and the distal part of the tibia, and held in a frame for external fixation of bone fractures (Standard fixation, Veterinary instrumentation, UK) at the required angle. The inclusion criterion for the radiographs was superimposition of the femoral condyles within a maximum tolerance of $2.0 \mathrm{~mm}$. The total number of radiographic images recorded in this study was 52 .

For radiological determination of the stifle angles, four different methods of landmarking were used and applied to the radiographs (Plate IV, Fig. 1). These were as follows:

\section{The goniometric method (GM)}

The long axis of the femur was defined as the line connecting the midpoint of the shaft in the minor trochanter region with the centre of the medial condyle of the femur. The centre of the condyle was defined with the Blumensaat's line, as a tangent drawn along the roof of the intercondylar notch and separating the articular surfaces of the condyles (Blumensaat 1938; Barnes and Owen 2015). The axis of the tibia was defined as a line connecting the midpoint of the most distal part of the tibia just above the tibiotarsal joint and the centre of the medial condyle of the femur.

The long axis method (LAM)

The long axes of the femur and tibia were defined based on the lines between the two shaft midpoints in the proximal and middle parts of the femoral length, and the two shaft midpoints in the distal and middle parts of the tibia. 
The eminence method (EM)

This was defined as the angle between the greater trochanter of the femur, the midpoint of the tibial intercondylar eminence and the midpoint of the most distal part of the tibia just above the tibiotarsal joint.

The kinematic method (KM)

This measured the angle between the greater trochanter of the femur, the midpoint of the distance between the distal sesamoid bone $\mathrm{m}$. gastrocnemii and the fibular head just caudal from the femoral condyles, and the midpoint of the most distal part of the tibia just above the tibiotarsal joint (Bush et al 2011).

All radiographic measurements were repeated two times with a three-month interval, by the same observer, who was appropriately trained and familiar with the stifle anatomy. For each of the two assessments, radiographs were presented in a random order to help ensure on the second occasion that the observer was unaware of their initial angle.

\section{Statistical analysis}

Measured values were expressed as means \pm standard deviations. One-way analysis of variance (ANOVA) was performed to compare groups with continuous data which were normally distributed according to the results of the Shapiro-Wilk normality test. Tukey's multiple comparison was used as the post hoc test for comparison of individual radiographic methods. For evaluation of differences between the RM and the angles physically set on the cadaver limbs, we used Dunnett's multiple comparisons test as the post hoc test. Significance determining the difference between the groups was considered for a $P$ value of 0.05 .

Intra-observer agreement was evaluated with the two-way random single measures intra-class correlation coefficient for absolute agreement (ICC 2,1) (Shrout and Fleiss 1979). Measurements were grouped by segregating the first and second rounds. The Intra-class correlation coefficient (ICC) ranged from 0 (no agreement) to 1 (perfect agreement). An ICC $<0.5$ was considered as indicating poor reliability, values between 0.5 and 0.75 indicated moderate reliability, values between 0.75 and 0.9 indicated good reliability, and values greater than 0.90 indicated excellent reliability (Koo and $\mathrm{Li}$ 2016).

All statistical analyses were performed using the IBM SPSS version 27 statistical software.

\section{Results}

A total number of 13 stifles were included in this study, and 52 radiographs were evaluated. Results are shown in Table 1 and are presented separately, divided into groups according to the angles set on the cadaver limbs.

Table 1. Mean values, standard deviations and 95\% confidence interval of individual radiographic methods shown separately according to angles set on cadaver limbs. Values are expressed in degrees.

\begin{tabular}{llrrrrrrr}
\hline $\mathrm{C}$ & \multicolumn{2}{l}{$\mathrm{GM}$} & \multicolumn{2}{c}{ LAM } & \multicolumn{2}{c}{ EM } & \multicolumn{2}{c}{ KM } \\
\cline { 2 - 9 } & $\mathrm{M} \pm \mathrm{SD}$ & $95 \% \mathrm{CI}$ & $\mathrm{M} \pm \mathrm{SD}$ & $95 \% \mathrm{CI}$ & $\mathrm{M} \pm \mathrm{SD}$ & $95 \% \mathrm{CI}$ & $\mathrm{M} \pm \mathrm{SD}$ & $95 \% \mathrm{CI}$ \\
\hline 125 & $132.4 \pm 5.0$ & $130.2-134.6$ & $126.4 \pm 4.5$ & $124.4-128.4$ & $143.3 \pm 4.2$ & $141.5-145.2$ & $146.7 \pm 4.9$ & $144.6-148.9$ \\
130 & $136.9 \pm 4.7$ & $134.8-139.0$ & $127.6 \pm 5.4$ & $127.6-132.5$ & $146.9 \pm 4.6$ & $144.9-148.9$ & $150.3 \pm 4.7$ & $148.2-152.4$ \\
135 & $141.0 \pm 5.2$ & $138.7-143.3$ & $133.3 \pm 6,3$ & $130.5-136.1$ & $150.6 \pm 6.1$ & $147.9-153.3$ & $154.2 \pm 6.6$ & $151.2-157.1$ \\
140 & $144.0 \pm 5.5$ & $141.5-146.4$ & $136.8 \pm 6.5$ & $133.9-139.6$ & $153.4 \pm 5.6$ & $150.9-155.9$ & $157.7 \pm 6.4$ & $154.9-160.5$ \\
\hline
\end{tabular}

M - mean value; SD - standard deviation; 95\% CI - confidence interval; C - Cadaver; GM- goniometric method; LAM - long axis method; EM - eminence method; KM - kinematic method. 
Table 2. Comparison of angles set physically on cadaver limbs with individual radiographic method results. Values are expressed in degrees.

\begin{tabular}{llcl}
\hline C & \multicolumn{1}{c}{ RM } & Mean difference & $P$ value \\
\hline \multirow{2}{*}{125} & C vs. GM & 7.44 & $<0.05$ \\
& C vs. LAM & 1.40 & $>0.05^{\mathrm{a}}$ \\
& C vs. EM & 18.35 & $<0.05$ \\
& C vs. KM & 21.74 & $<0.05$ \\
\hline \multirow{3}{*}{130} & C vs. GM & 6.90 & $<0.05$ \\
& C vs. LAM & 0.04 & $>0.05^{\mathrm{a}}$ \\
& C vs. EM & 16.91 & $<0.05$ \\
& C vs. KM & 20.31 & $<0.05$ \\
\hline \multirow{3}{*}{135} & C vs. GM & 6.01 & $<0.05$ \\
& C vs. LAM & 1.67 & $>0.05^{\mathrm{a}}$ \\
& C vs. EM & 15.62 & $<0.05$ \\
& C vs. KM & 19.18 & $<0.05$ \\
\hline \multirow{2}{*}{140} & C vs. GM & 3.95 & $>0.05^{\mathrm{a}}$ \\
& C vs. LAM & 3.24 & $>0.05^{\mathrm{a}}$ \\
& C vs. EM & 13.43 & $<0.05$ \\
& C vs. KM & 17.69 & $<0.05$ \\
\hline
\end{tabular}

C - angle set on cadaver limb; RM - radiographic method; GM - goniometric method; LAM - long axis method; EM - eminence method; KM - kinematic method; ${ }^{\text {a }}$ - nonsignificant difference

Significant differences were observed comparing the measured angles between most RM $(P<0.05)$ in each angle group, except between the EM and KM (Table 3, Fig. 2).

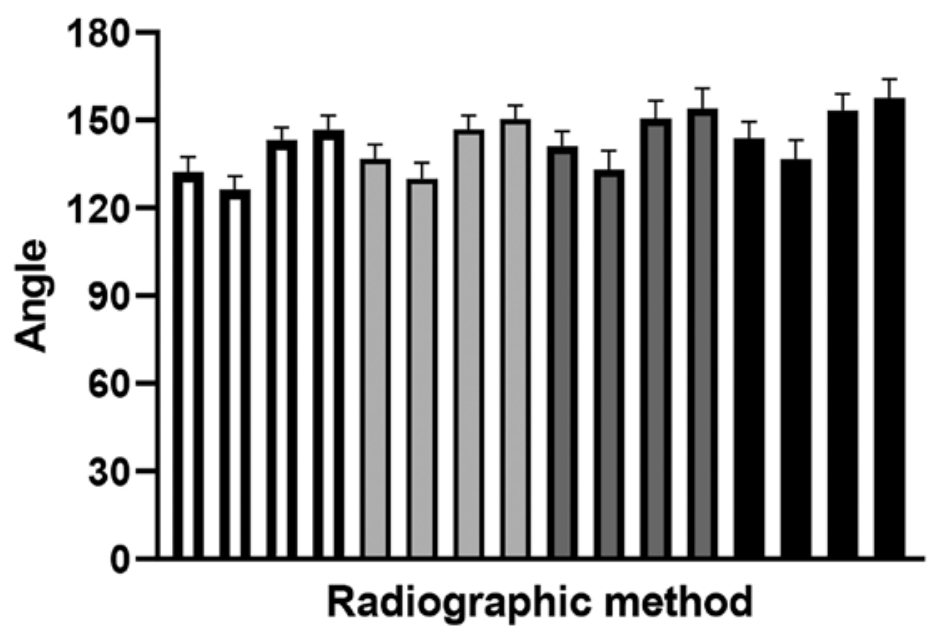

Fig. 2. Mean values and standard deviations of radiographic methods.

Different colours represent individual angles set on cadaver limbs: white $-125^{\circ}$, light grey - $130^{\circ}$, dark grey $-135^{\circ}$, black $-140^{\circ}$. Columns from left to right represent radiographic methods: 1) goniometric method, 2) long axis method, 3) eminence method, 4) kinematic method.
Comparisons of angles set on cadaver limbs and individual radiographic measurements are shown in Table 2. We found no significant differences between angles set on the cadaver limbs and angles measured by the LAM in any angle group, with a mean difference of $1.4^{\circ}$ $(P=0.62)$ in the $125^{\circ}$ group; $0.04^{\circ}$ $(P=0.99)$ in the $130^{\circ}$ group; $1.67^{\circ}$ $(P=0.69)$ in the $135^{\circ}$ group, and $3.24^{\circ}(P=0.15)$ in the $140^{\circ}$ group. Significant differences were found between angles set on the cadaver limbs and angles measured by the GM in three groups, with a mean difference of $7.44^{\circ}$ in the $125^{\circ}$ group; $6.9^{\circ}$ in the $130^{\circ}$ group; $6.01^{\circ}$ in the $135^{\circ}$ group; there was no significant difference in the $140^{\circ}$ group with a mean difference of $3.95^{\circ}(P=0.06)$. The two other methods (EM and KM) differed significantly from the angles set on the cadaver limbs in all four angle groups $(P<0.05)$

\section{Radiographic method}


Table 3. Comparison of individual radiographic methods in each group of measurements based on different angles fixed on cadaver limbs. Values are expressed in degrees.

\begin{tabular}{|c|c|c|c|}
\hline $\mathrm{C}$ & $\mathrm{Rm}$ & Mean difference & $P$ value \\
\hline \multirow{6}{*}{125} & GM vs. LAM & 6.03 & $<0.05$ \\
\hline & GM vs. EM & 10.91 & $<0.05$ \\
\hline & GM vs. KM & 14.30 & $<0.05$ \\
\hline & LAM vs. EM & 16.94 & $<0.05$ \\
\hline & LAM vs. KM & 20.33 & $<0.05$ \\
\hline & EM vs. KM & 3.38 & $>0.05^{\mathrm{a}}$ \\
\hline \multirow{6}{*}{130} & GM vs. LAM & 6.85 & $<0.05$ \\
\hline & GM vs. EM & 10.00 & $<0.05$ \\
\hline & GM vs. KM & 13.40 & $<0.05$ \\
\hline & LAM vs. EM & 16.86 & $<0.05$ \\
\hline & LAM vs. KM & 20.26 & $<0.05$ \\
\hline & EM vs. KM & 3.40 & $>0.05^{\mathrm{a}}$ \\
\hline \multirow{6}{*}{135} & GM vs. LAM & 7.68 & $<0.05$ \\
\hline & GM vs. EM & 9.61 & $<0.05$ \\
\hline & GM vs. KM & 13.17 & $<0.05$ \\
\hline & LAM vs. EM & 17.29 & $<0.05$ \\
\hline & LAM vs. KM & 20.85 & $<0.05$ \\
\hline & EM vs. KM & 3.55 & $>0.05^{\mathrm{a}}$ \\
\hline \multirow{6}{*}{140} & GM vs. LAM & 7.19 & $<0.05$ \\
\hline & GM vs. EM & 9.47 & $<0.05$ \\
\hline & GM vs. KM & 13.73 & $<0.05$ \\
\hline & LAM vs. EM & 16.67 & $<0.05$ \\
\hline & LAM vs. KM & 20.93 & $<0.05$ \\
\hline & EM vs. KM & 4.25 & $>0.05^{\mathrm{a}}$ \\
\hline
\end{tabular}

$\mathrm{C}$ - angle set on cadaver limb; RM - radiographic method; GM - goniometric method; LAM - long axis method; EM - eminence method; KM - kinematic method; ${ }^{a}$ - nonsignificant difference was 0.06 , which was almost a significant difference. Another ex vivo study in which the authors evaluated the reliability of goniometry by comparing goniometric measurements with radiographic measurements found no significant differences between goniometric and radiographic measurements (Jaegger et al. 2002). In the study, the authors did not define the exact point of the condyle at which they made their radiographic measurements, and they used the greater trochanter for the femoral axis, which was not what we did. In addition, the radiographic measurements were done by one observer only once. To our knowledge, only one study has used the Blumensaat's line to measure stifle angles in dogs. The authors have reported that the Blumensaat's line provided a useful consistent radiological landmark on the distal femur for the assessment of stifle joint angulation (Barnes and Owen 2015). Our intra-observer measurements (ICC Gm- 0.97) confirmed that the Blumensaat's line is a replicable and constant feature for radiographic measurements. The angles set on the cadaver limbs using a goniometer differed significantly from the radiographic measurements using the GM. One of the possible reasons for this difference is that we measured the angles on the cadaver limbs still bearing the soft tissues, which may have led to slightly inaccurate locating of the centre of the medial epicondyle. The lastly mentioned study was conducted on client-owned dogs, and the authors did not use a goniometer but a neutral stifle angle 
(nSA) for radiographic positioning, which was physiological for each particular dog. In addition, they used the mechanical axis of the femur rather than the mid-shaft line as we did, so the results cannot be compared.

We did not find significant differences between the angles set on cadaver limbs and the LAM in any group. Significant differences were found between angles set on cadaver limbs and angles measured by the GM in three groups but was no significant difference was found in the $140^{\circ}$ group, while the other methods differed significantly in all groups. By comparing individual RM, we found significant differences between all of them, except between the EM and KM. This finding almost completely supports the hypothesis that using different radiographic landmark methods would result in different stifle angles, which may subsequently affect the calculated advancements of some tibial osteotomies. According to the findings of another study, the use of different anatomical landmark methods for stifle angle determination can also result in different angles, and finally influence the value of the required advancement of tuberositas tibiae in the TTA procedure. The study did not identify the clinically relevant TTA required to achieve a PTA of $90^{\circ}$ using the EM and KM methods, only greater advancement with the LAM (Bush et al. 2011). Our results are similar, with large discrepancies found between the LAM vs. EM and KM but no significant difference between the EM and KM.

There are many considerations regarding the pre-planning of TTA, but the aim of each plan is to achieve a perpendicular post-operative PTA (Kapler et al. 2015). Some of these methods require the joint to be set close to full extension, at approximately $135^{\circ}$, which is the average midstance stifle angle at the walk and trot, as determined in kinematic gait studies with well-defined landmarks (Allen et al. 1994; Schaefer et al. 1998; Tepic et al. 2002; Kipfer et al. 2008; Hoffmann et al. 2011). In contrast, the landmarks used for measuring stifle angle in ex vivo clinical studies reporting the TTA surgical procedure are not described (Apelt et al. 2007; Miller et al. 2007; Kim et al. 2009). Consequently, during TTA planning, if the stifle is positioned at $135^{\circ}$ using inappropriate landmarks, the resultant stifle angle may actually be more flexed or extended than the required $135^{\circ}$ angle determined in kinematic studies. This situation can affect the PTA, because stifle joint flexion is a major contributor to the PTA, with a linear decrease in the PTA with increasing flexion (Dennler et al. 2006). The landmarks for angle measurement, if they are described, vary among the studies (Jaegger et al. 2002; Dennler et al. 2006; Lafaver e al. 2007; Voss et al. 2008; Hoffmann et al. 2011; Millet et al. 2013; Cadmus et al. 2014). If the stifle is not at a true $135^{\circ}$ angle because of a discrepancy between the methods of angle measurement, or the midstance angle of $135^{\circ}$ cannot be applied to all dogs, then radiographs from standing dogs should be made to determine the real neutral angle of the joint. Pre-operative measuring using inappropriate radiographs may result in under- or over-advancement of the tibial tuberosity. Residual cranial tibial translation is a potentially clinically important post-operative finding after TTA. The high number of meniscal tears associated with TTA in the initial phase of clinical introduction may be due, at least in part, to residual cranial translation. Numerous theories have been proposed for under-advancement during TTA (Meeson et al. 2018). A recent study reported that the selection of a larger cage size during TTA may be advantageous to compensate for under-advancement and to minimize the risk of residual cranial tibial translation (Jin et al. 2018). In light of another recent study which showed that $70 \%$ of TTA dogs had persistent post-operative femorotibial subluxation on weight-bearing radiographs, any combination of methods that would lead planners to select a larger advancement cage size may be warranted (Skinner et al. 2013). One of the possible reasons for the results of these studies is an inaccurate pre-operative assessment due to incorrectly measured angles in the stifles, as the procedure for measuring the joint angle is not precisely defined and the available methods provide different results. 
A single observer was responsible for all measurements in this study, but for that reason they were done twice. Determination of the Blumensaat's line is subjective, although it is a distinct landmark and there should not be a wide inter-observer variation in its assessment. The limbs of large dogs of various breeds were used in this study, whereas there may be differences between the angles in the stifle joint of small breeds due to differences in their bone formation.

In conclusion, we found large discrepancies between the angles set physically on the cadaver limbs and the radiographic measurements, except for the LAM, and also between individual RM, except EM and KM. These results suggest that using different radiographic landmark methods results in different stifle angles, which may have a negative impact on further measurements. The specific stifle angle measurement method should be considered when comparing similar studies, and also in pre-operative measurements for some tibial osteotomies aimed at stabilizing the joint.

\section{Acknowledgement}

This study was supported by the Ministry for Education, Science, Research and Sport of the Slovak Republic and the Slovak Academy of Sciences through VEGA project no. 1/0561/19.

\section{References}

Allen K, DeCamp CE, Braden TD, Balms M 1994: Kinematic gait analysis of the trot in healthy mixed breed dogs. Vet Comp Orthop Traumatol 7: 148-153

Apelt D, Kowaleski MP, Boudrieau RJ 2007: Effect of tibial tuberosity advancement on cranial tibial subluxation in canine cranial cruciate-deficient stifle joints: an in vitro experimental study. Vet Surg 36: 170-177

Barnes DC, Owen MR 2015: Indirect assessment of stifle angle for improved accuracy of preoperative planning of tibial osteotomy procedures in dogs. Vet Record 177: 99-99

Blumensaat C 1938: Die Lageabweichungen und Verrenkungen der Kniescheibe. In: Payr E, Kirschner M (Eds): Ergebnisse der Chirurgie und Orthopädie. Berlin, Heidelberg: Springer, pp. 149-223

Bush MA, Bowlt K, Gines JA, Owen MR 2011: Effect of use of different landmark methods on determining stifle angle and on calculated tibial tuberosity advancement. Vet Comp Orthop Traumatol 24: 205-210

Cadmus J, Palmer RH, Duncan C 2014: The effect of preoperative planning method on recommended tibial tuberosity advancement cage size. Vet Surg 43: 995-1000

Dennler R, Kipfer NM, Tepic S, Hassig M, Montavon PM 2006: Inclination of the patellar ligament in relation to flexion angle in stifle joints of dogs without degenerative joint disease. Am J Vet Res 67: 1849-1854

Hoffman DE, Miller JM, Ober CP, Lanz OI, Martin RA, Shires PK 2006: Tibial tuberosity advancement in 65 stifles. Vet Comp Orthop Traumatol 19: 219-227

Hoffmann DE, Kowaleski MP, Johnson KA, Evans RB, Boudrieau RJ 2011: Ex vivo biomechanical evaluation of the canine $\mathrm{CrCL}$ deficient stifle with varying angles of stifle joint flexion and axial loads after TTA. Vet Surg 40: $311-320$

Jaegger G, Marcellin-Little DJ, Levine D 2002: Reliability of goniometry in Labrador Retrievers. AJVR 63: 979-986

Jin DW, Peck JN, Tano CA, Morgan MJ 2018: Discrepancy between true distance of tibial tuberosity advancement and cage size: An ex vivo study. Vet Surg 48: 186-191

Kapler MW, Marcellin-Little DJ, Roe SC 2015: Planned wedge size compared to achieved advancement in dogs undergoing the modified Maquet procedure. Vet Comp Orthop Traumatol 28: 379-384

Kim SE, Pozzi A, Banks SA, Conrad BP, Lewis DD 2009: Effect of tibial tuberosity advancement on femorotibial contact mechanics and stifle kinematics. Vet Surg 39: 33-39

Kipfer NM, Tepis S, Damur DM, Guerrero T, Hässig M, Montavon PM 2008: Effect of tibial tuberosity advancement on femorotibial shear in cranial cruciate-deficient stifles: an in vitro study. Vet Comp Orthop Traumatol 21: $385-390$

Koo TK, Li MY 2016: A guideline of selecting and reporting intraclass correlation coefficients for reliability research. J Chiropr Med 15: 155-163

Lafaver S, Miller NA, Stubbs WP, Taylor RA, Boudrieau RJ 2007: Tibial tuberosity advancement for stabilization of the canine cranial cruciate ligament-deficient stifle joint: surgical technique, early results, and complications in 101 dogs. Vet Surg 36: 573-586

Meeson RL, Corah L, Conroy MC, Calvo I 2018: Relationship between tibial conformation, cage size and advancement achieved in TTA procedure. Vet Res 14: 104

Miller JM, Shires PK, Lanz OI, Martin RA, Grant JW 2007: Effect of $9 \mathrm{~mm}$ tibial tuberosity advancement on cranial tibial translation in the canine cranial cruciate ligament deficient stifle. Vet Surg 36: 335-340 
Millet M, Bismuth C, Labrunie A, Marin B, Filleur A, Pillard P, Sonet J, Cachon T, Etchepareborde S 2013: Measurement of the patellar tendon tibial plateau angle and tuberosity advancement in dogs with cranial cruciate ligament rupture. Vet Comp Orthop Traumatol 26: 469-478

Montavon PM, Damur DM, Tepic S 2002: Advancement of the tibial tuberosity for the treatment of cranial cruciate deficient canine stifle. In Proceedings of the $1^{\text {st }}$ World Orthopaedic Veterinary Congress, Munich, Germany, September 5-8, 152 (Abstract)

Nisell R, Németh G, Ohlsén H 1986: Joint forces in the extension of the knee: analysis of a mechanical model. Acta Orthop Scand 57: 41

Schaefer SL, DeCamp CE, Hauptman JG, Walton A 1998: Kinematic gait analysis of hind limb symmetry in dogs at the trot. Am J Vet Res 59: 680-685

Shrout PE, Fleiss JL 1979: Intraclass correlations: uses in assessing rater reliability. Psychol Bull 86: 420-428

Skinner OT, Kim SE, Lewis DD, Pozzi A 2013: In vivo femorotibial subluxation during weight-bearing and clinical outcome following tibial tuberosity advancement for cranial cruciate ligament insufficiency in dogs. Vet J 196: 86-91

Stein S, Schmoekel H 2008: Short-term and eight to 12 months results of a tibial tuberosity advancement as treatment of canine cranial cruciate ligament damage. J Small Anim Pract 49: 398-404

Tepic S, Damur DM, Montavon PM 2002: Biomechanics of the stifle joint. In Proceedings of the $1^{\text {st }}$ World Orthopaedic Veterinary Congress, Munich, Germany, September 5-8, 2002 189-190 (Abstract)

Tepic S, Montavon PM 2004: Is cranial tibial advancement relevant in the cruciate deficient stifle? In Proceedings of the $12^{\text {th }}$ ESVOT Congress, Munich, Germany, September 10-12, 2004 (Abstract)

Vasseur PB 2003: Stifle joint. In: Slatter D (Ed): Textbook of Small Animal Surgery. Third edn. Philadelphia, WB Saunders Co, 2896 p.

Voss K, Damur DM, Guerrero T, Haessig M, Montavon PM 2008: Force plate gait analysis to assess limb function after tibial tuberosity advancement in dogs with cranial cruciate ligament disease. Vet Comp Orthop Traumatol 21: 243-249 
Plate IV

Ševčík K. et al.: Radiographic ... pp. 399-406

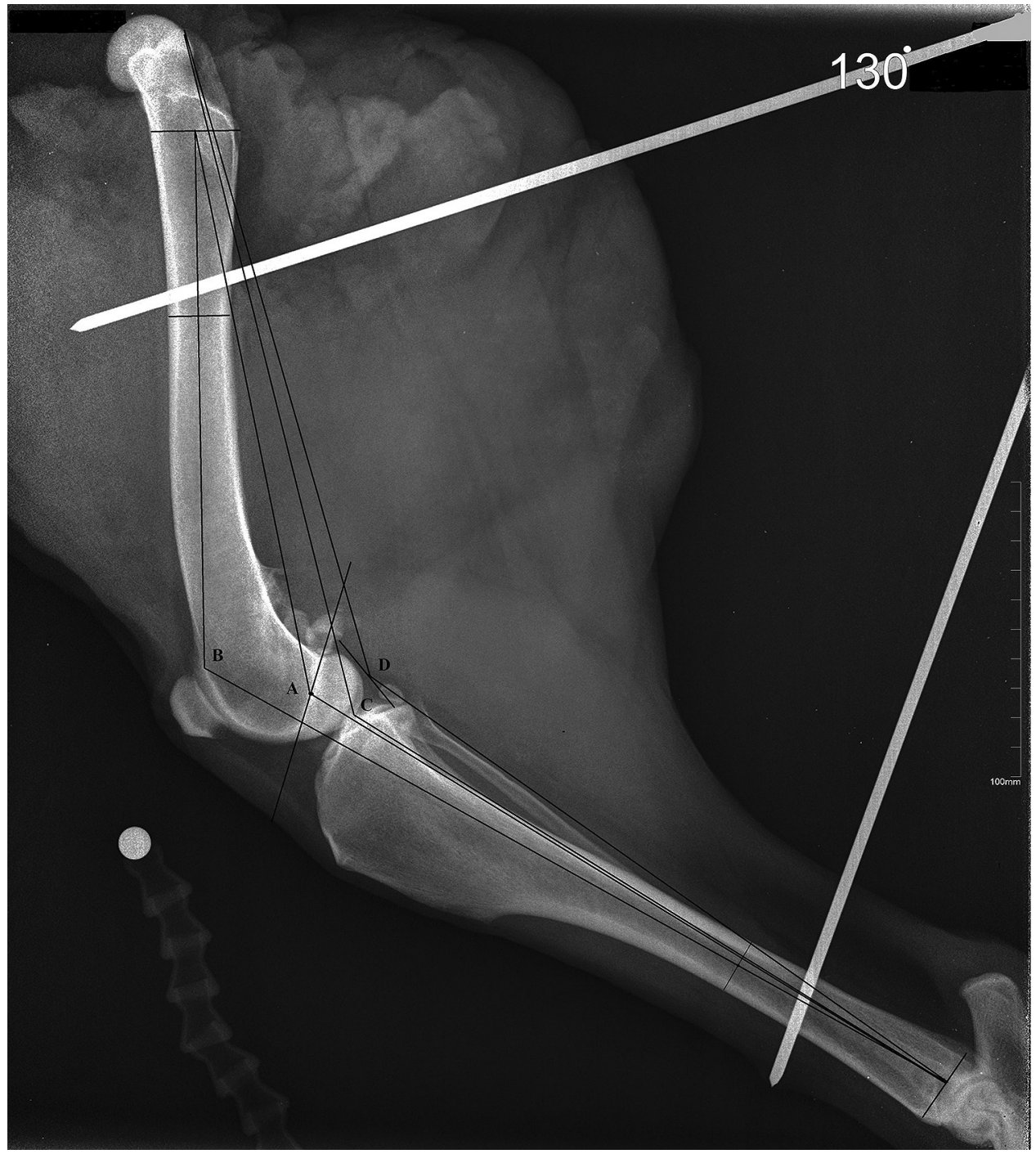

Fig. 1. Radiographic representation of landmarks used to determine the stifle angle. The stifle joint is fixed at $130^{\circ}$. A - goniometric method, B - long axis method, C - eminence method, D - kinematic method 